Astronomical images are intrinsically blurred because of diffraction at the edges of the telescopes' optics. Although increasing the aperture size reduces this limitation, the images so produced in practice are merely brighter. Their resolution is no better than that from a telescope $10-20 \mathrm{~cm}$ in diameter $(0.5-1.0$ arcseconds, equivalent to a resolution of $2.5-5 \mathrm{~mm}$ at $1 \mathrm{~km}$ ) owing to the atmospheric spoiling. This waste of potential resolving power will become greater with the next generation of telescopes with apertures as large as $10 \mathrm{~m}$.

Adaptive optics, however, allows one to correct all aberrations the lightwave encounters on its way from space to the astronomical detector. A wavefront sensor measures the optical distortions which can then be nullified by deforming a flexible mirror in the telescope. To be effective, the corrections have to be made up to several hundred times per second, depending on the observing wavelength and atmospheric conditions. The result will then be diffractionlimited images as if there were no atmosphere above the telescope.

Babcock's proposal seemed to be forgotten until the early 1970 s, at which time the propagation of laser radiation through the atmosphere became of military interest, notably in the Strategic Defense Initiative (SDI or 'starwars'). But while that research continued in secret, astronomers considered the solu- tion to their difficulties lay in going into space. The Hubble Telescope is the crowning achievement of that approach. Only in the past 8-10 years has progress been made in the nonmilitary use of adaptive optics. The first successful tests were performed in 1989, using telescopes operating in the infrared wavelength range $^{4,5}$ (Fig. 2). But although the technology already existed in the SDI
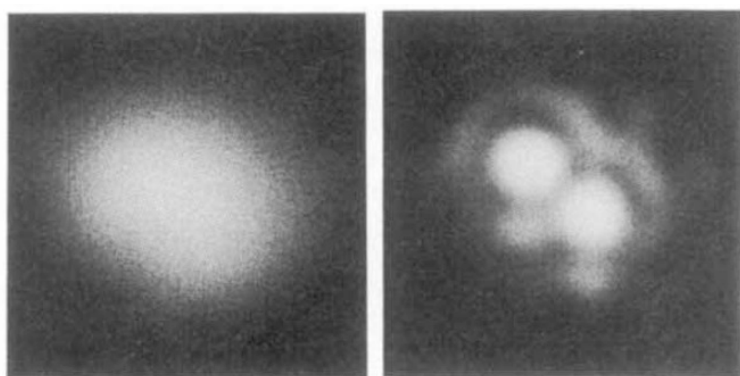

FIG. 2 A distinct improvement: by adjusting their telescope's optics, ESO astronomers nullified atmospheric distortion to obtain a clear image of the binary star HR6658.

laboratories, most of it had to be reinvented, owing to classification.

In any case, adaptive optics is not so easy to apply to astronomical imaging. To make any correction, one must first measure the error. Most interesting astronomical objects are too faint for correction with adaptive optics. In some cases, there are bright stellar objects nearby in the field of view. These can be used in measurement of the disturbed wavefront if their light travels through the same atmospheric layers. In the infrared range, at wavelengths greater than $5 \mu \mathrm{m}$, the whole sky is covered by sufficiently bright objects, because the reference need not be so close to the target object. But for shorter wavelengths, and, in particular, in the visible range, only a small fraction of the sky is suitably covered.
In 1985, the French astronomers Foy and Labeyrie proposed ${ }^{6}$ the futuristic technique of artificial stars to overcome 1987 seemed to indicate its feasibility ${ }^{7}$. The artificial star is generated by focusing a powerful laser beam in a distinct layer of the atmosphere. Rayleigh scattering in the lower atmosphere or resonance scattering in the mesospheric sodium layer at $80-100 \mathrm{~km}$ then creates a bright point of light which is used in wavefront measurement. The astronomical imaging and wavefront sensing processes have to be rapidly alternated to avoid polluting the image detector with light from the artificial star.

What was considered a dream by many astronomers now turns out to be reality. Indeed, the first classified tests in 1983 demonstrated the feasibility of this shortage of reference stars. Tests in

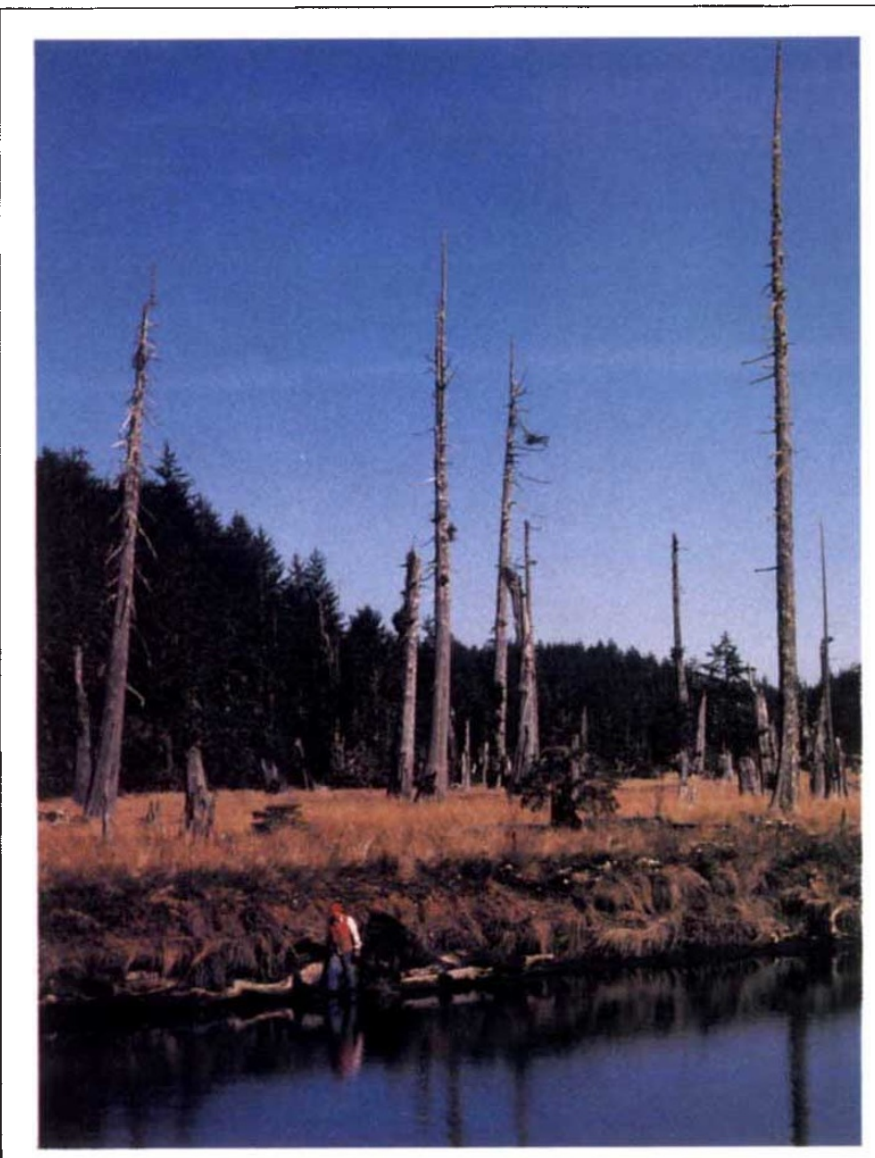

\title{
Only one earthquake
}

GHost forests of western red cedar along the coast of Washington state bear witness to a cataclysmic earthquake that struck about 300 years ago. They also bear a reminder for the 10 million inhabitants in the region of the future devastation that may threaten there. Tectonic convergence at the Cascadia subduction zone along the coast of northern California, Oregon, Washington and Vancouver Island is proceeding at a rate of $2.5-4.5 \mathrm{~cm} \mathrm{yr} r^{-1}$, comparable to the convergence at the seismically active Nankai trough off southwest Japan, yet the zone is completely quiescent. Evidence such as the ghost forests suggests that the Pacific and North American plates are rigidly locked and accumulate enormous amounts of strain that is released episodically in massive earthquakes. The last such is dated by B. F. Atwater and colleagues on page 158 of this issue to the years $1680-1720$. This date, 60 years or more following the European colonization of Massachusetts, comes as no surprise to those following developments in the study of prehistoric earthquakes along the west coast of North America. The real achievement of Atwater et al. is to show, through impressively precise radiocarbon carbon dating of the timber, that two forests separated by $55 \mathrm{~km}$ perished at the same time, owing to sudden subsidence of coastal lowlands and immersion of the trees in salt water. From this it seems that a single powerful earthquake was responsible, not several smaller ones. If the authors can extend their results to ghost forests $100 \mathrm{~km}$ or more apart, it would show that the last event was a 'great' earthquake of magnitude 8-9 - almost as powerful as the one that rocked Chile in 1960. The comparison is not idle: the Chilean earthquake stirred up 10-m tsunami waves that left coastal sand deposits similar to ones found along the Pacific northwest.

Roland Pease 